\title{
Exposição à violência entre adolescentes de uma comunidade de baixa renda no Nordeste do Brasil
}

\author{
Exposure to violence among adolescents \\ in a low-income community in the northeast of Brazil
}

Deborah Pedrosa Moreira ${ }^{1}$

Luiza Jane Eyre de Souza Vieira ${ }^{2}$

Augediva Maria Jucá Pordeus ${ }^{2}$

Samira Valentim Gama Lira ${ }^{2}$

Geisy Lanne Muniz Luna ${ }^{2}$

Juliana Guimarães e Silva ${ }^{3}$

Maria de Fátima Antero Sousa Machado ${ }^{2}$

${ }^{1}$ Centro de Educação Permanente em Vigilância da Saúde, Escola de Saúde Pública do Ceará. Av. Antônio Justa 3161,

Meireles. 60165-090

Fortaleza CE.

deborahpm@gmail.com

${ }^{2}$ Mestrado em Saúde

Coletiva, Centro de

Ciências da Saúde,

Universidade de Fortaleza.

${ }^{3}$ Escola Nacional de Saúde

Pública, Fiocruz.

\begin{abstract}
This a cross-sectional study made in Fortaleza, Ceará, 2009, which included 458 teenagers and analyzed their exposure to violence, describing their access to weapons, alcohol abuse, illegal drug use and their self-esteem by investigating their socio-economic, school and family characteristics and exposure to the phenomenon. A questionnaire and/or structured interviews were used for data collection, and analysis involved Pearson's chi-square test, with 95\% reliability. Of the 458 participants, $17.7 \%$ were considered to be exposed to criminal violence. Significant variables for exposure to violence included: place of birth $(p=0.020)$, years of schooling ( $p=$ $0,009)$, school absenteeism $(p<0.001)$, the father as the head of the family $(p=0.026)$, alcoholaddicted parents $(p<0.001)$, good/very good family relationships $(p=0.009)$, and parents' dissatisfaction with their children's friends $(p<0.001)$. Thus, it is necessary that public policies focus on a support network for care of adolescents and that urban centers organize themselves socially and politically in the quest for understanding the effects of exposure to violence among adolescents in low-income communities.
\end{abstract}

Key words Adolescence, Violence, Risk factors, Family characteristics, Cross-sectional studies, Social condition
Resumo O estudo analisou a exposição dos adolescentes à violência, considerando o acesso à arma, o uso abusivo de álcool elou uso de drogas ilícitas e sua autostima, e investigou a influência de fatores socioeconômicos, escolares e características familiares com a exposição a esse fenômeno. Estudo transversal, realizado em Fortaleza, Ceará, em 2009, com 458 adolescentes. Foram utilizados questionários elou entrevistas estruturadas para coleta dos dados e na análise aplicamos o teste de correlação de Pearson, com a confiabilidade de 95\%. Ao correlacionar a exposição do adolescente à violência com as variáveis naturalidade ( $p=0,020$ ), tempo de estudo em anos ( $p=0,009)$, absenteísmo escolar $(p<0,001)$, responsável financeiro pela família ( $p=0,007$ ), pais ou responsáveis etilistas $(p<0,001)$, relações familiares boas/muito boas $(p=0,009)$ e a não satisfação dos pais com amizades de seus filhos ( $p<0,001$ ), identificamos associação direta. Assim, é necessário que as políticas públicas enfoquem rede de apoio ao cuidado como adolescente e que os centros urbanos organizem-se social e politicamente na busca pela compreensão dos efeitos da exposição à violência em adolescentes de comunidades de baixa renda.

Palavras-chave Adolescência, Violência, Fatores de risco, Características familiares, Estudos Transversais, Condição Social 


\section{Introdução}

Os efeitos traumáticos ocasionados pela violência na vida da criança e adolescente trouxeram atenção a este problema de saúde pública que assola incessantemente a sociedade ${ }^{1}$. Pesquisas sobre violências reafirmam a evidência e continuísmo desse fenômeno no cotidiano das cidades e o envolvimento crescente de jovens nesses eventos, ora como vítimas, ora como autores ${ }^{2,3}$, o que suscita a importância de continuar com investigações nessa temática.

A literatura sinaliza que a experiência do adolescente com situações de violência proporciona mudanças de atitudes, com perspectiva de o indivíduo desenvolver um comportamento violento ${ }^{4}$. Essa predisposição instiga os pesquisadores do tema a analisar os direitos e deveres, as ações sociais e as políticas públicas destinadas aos adolescentes $^{5}$ no sentido de minimizar e desenvolver estratégias de prevenção da violência neste grupo.

A violência contra o adolescente ganhou visibilidade por meio dos estatutos, leis e políticas públicas, bem como da constituição de conselhos tutelares, mas apesar dos diversos documentos e instituições voltadas para a assistência ao adolescente, identifica-se a falta de articulação na busca de formação de redes de apoio. Assim, na prevenção da violência urgem a reflexão e a mobilização da sociedade e dos profissionais envolvidos na assistência ao adolescente ${ }^{6}$ vitimado pelas violências.

Desta forma, os profissionais devem ser conhecedores de fatores que se associam à violência, como a desagregação familiar, uso indevido de tempo, desintegração de valores tradicionais, influência de amizades e marginalização social, pois estes colaboram para que o adolescente reconstrua sua identidade nos novos espaços sociais, servindo como ferramenta preventiva ${ }^{1,3,6}$.

A realização de medidas preventivas durante a adolescência é um desafio, pois é a fase da vida que impõe transformações e interferências do meio familiar e social. Essa etapa faz com que o adolescente tente se rebelar contra a realidade vivenciada ${ }^{7}$, como usar drogas lícitas e ilícitas, ter acesso a armas e conviver com familiares que usam drogas e reproduzem a violência no contexto familiar. Este conjunto disfuncional proporciona a exposição do adolescente à violência ${ }^{8-10}$.

O envolvimento/exposição desse grupo com as tipologias da violência, nos últimos anos, custou ao governo investimentos em programas e políticas que favorecem a "construção" de uma adolescência saudável ${ }^{3,6}$. Por outro lado, existem lacunas de estudos que busquem identificar a associação dos fatores como uso de armas, uso de álcool e/ou drogas ilícitas e características familiares com a exposição do adolescente à violência na comunidade. Na literatura nacional e internacional $^{11-15}$, encontramos pesquisas envolvendo adolescentes e violência, mas centrada no adolescente em conflito com a lei ou a violência delinquencial, que esquiva do proposto neste estudo.

No intuito de contribuir com esta lacuna, o estudo (i) analisou a exposição dos adolescentes à violência, considerando o acesso à arma, o uso abusivo de álcool e/ou uso de drogas ilícitas e a autoestima dos adolescentes, e (ii) investigou a influência de fatores socioeconômicos, escolares e características familiares com a exposição a esse fenômeno.

\section{Métodos}

Trata-se de um estudo transversal no qual foram selecionados 458 adolescentes de ambos os sexos, com idade mínima de dez e máxima de dezenove anos, residentes em uma comunidade de baixa renda em Fortaleza, Ceará.

A população de base deste estudo foi oriunda de um censo realizado nesta localidade em 2007/ 2008, com registro de 10.900 habitantes. Desta população, 2.300 eram adolescentes representando $21,0 \%$ da população total. A amostra foi estratificada por sexo e idade, correspondendo a 120 homens e 120 mulheres de $10-14$ anos (1 $1^{\mathrm{a}}$ fase da adolescência) e 120 homens e 120 mulheres de 15-19 anos ( $2^{\text {a }}$ fase da adolescência $)^{16}$. Porém, em virtude da dificuldade de encontrar os adolescentes em seus domicílios, foram aplicados 458 questionários ou entrevistas, abrangendo $95,4 \%$ da amostra.

A estratificação da amostra se deu em dois estágios: (i) seleção das ruas da comunidade, conforme censo e (ii) seleção dos domicílios para participar da coleta. Esta última, aleatória, foi escalada por alternância de casas (1/1), no trajeto esquerdo-direito no sentido horário. Em domicílios onde havia mais de um adolescente, todos participaram.

Para este estudo, foram critérios de inclusão: adolescente residir nesta comunidade e atender a faixa etária de 10 a 19 anos. Excluíram-se os adolescentes que informaram ter participado do teste piloto.

Em virtude de realização anterior do censo nessa comunidade (fase 0 ), a coleta de dados, realizada entre julho e outubro de 2009 , incluiu 
duas modalidades: (i) entrevista estruturada face a face para analfabeto funcional ${ }^{17}$ e (ii) questionário anônimo, com questões de múltipla escolha sobre fatores individuais, familiares, socioeconômicos e comunitários, com duração média de respostas de 30 minutos.

Importante explicitar conceitos analíticos do estudo: (i) família nuclear ou tradicional - consiste em um marido, uma esposa e seus filhos que vivem em um domicílio comum; (ii) família reconstituída - pais que se separaram, recasaram e constituíram novas uniões nucleares, ou seja, pelo menos um dos adultos é um padrasto ou uma madrasta; (iii) família monoparental a família de pai/mãe solteiro; (iv) família ampliada, estendida ou extensa - compõe-se da família nuclear dos membros da família de origem como os avós, tios, primos ${ }^{18}$.

Para a apreciação das variáveis, empregou-se a escala de autoestima de Rosenberg (1965) e o instrumento CAGE para análise do uso abusivo de álcool. No que se refere aos fatores de risco à violência, os mesmos foram orientados pelo Modelo Ecológico.

Para a identificação da autoestima, a Escala de Autoestima (AE) de Rosenberg (1965), adaptada e validada no Brasil ${ }^{19}$, adota a terminologia autoestima positiva e negativa. As pontuações menores que 25 representam autoestima negativa. Neste estudo, para efeito de análise, os questionários em que pelo menos um item não tinha sido respondido não foram validados.

$\mathrm{O}$ instrumento usado na pesquisa sobre o comportamento de risco dos estudantes adolescentes do estado do Ceará ${ }^{20}$, realizada com 11.701 participantes, orientou a mensuração das variáveis individuais, familiares e escolares, uso de drogas ilícitas e exposição de arma de fogo e/ou branca.

Quanto à renda familiar, os dados foram agrupados em categorias ampliadas (Renda menor que dois SM e renda maior ou igual a dois $\mathrm{SM})$. Quanto às relações familiares, o estudo considerou as alternativas mais expressivas: boas/ muito boas e ruins/muito ruins. Para analisar o uso abusivo de álcool, adaptamos o instrumento "CAGE"21 que estima a magnitude do alcoolismo em populações, e consta de quatro questões básicas a respeito da ingestão de álcool: $\mathrm{C}$ (cut-down - diminuir a ingestão), A (annoyed — irritado), G (guilty — culpado), E (eyeopener - identificação de ressaca), diante disso considerou-se uso abusivo de álcool respostas afirmativas a duas ou mais perguntas, e, alto risco, a uma pergunta.
Os fatores de risco à violência foram orientados pelo Modelo Ecológico ${ }^{22}$ nos níveis individual, relacional, comunitário e social. Neste modelo, o nível biológico identifica os fatores históricos biológicos e pessoais que a pessoa traz em seu comportamento, concentrando-se nas características que aumentam a possibilidade do indivíduo ser vítima ou perpetrador de violência. $\mathrm{O}$ estudo considerou as variáveis gostar de ir à escola e autoestima pertencentes ao nível biológico. O nível relacional considera as relações sociais próximas evidenciando-se as interações sociais, nos âmbitos mais próximos dos companheiros, dos colegas, dos parceiros íntimos, dos membros da família e sua influência na vitimização ou na perpetração da violência. Neste foco, as variáveis estudadas neste nível foram: relações familiares, satisfação dos pais com as amizades dos filhos e com o rendimento escolar.

No nível comunitário são nomeados os locais de trabalho, a escola e a vizinhança, e como problemas, os altos níveis de desemprego, a presença de tráfico de drogas e de armas e componentes de ordem relacional, como o isolamento social em que vivem determinadas famílias. Neste sentido, considera-se a renda familiar, o responsável pelo sustento da família trabalhando, absenteísmo escolar, acesso à arma, uso abusivo de drogas ilícitas e álcool e etilismo dos pais.

O nível social analisa os fatores sociais mais amplos que influenciam nos índices da violência, como normas culturais que justificam a violência como forma de resolver conflitos; atitudes que apreciam a opção pelo suicídio como um direito de escolha individual; machismo e cultura adultocêntrica, dentre outros. Neste caso, elencaramse para efeito analítico as variáveis: tipos de família, pais separados, responsável pela família.

Para este estudo, consideraram-se como expostos (Figura 1) os adolescentes que, em algum momento, tiveram exposição a uso abusivo de álcool ${ }^{21}$ e/ou uso de drogas ilícitas e, pelo menos, um dos seguintes fatores: (i) autoestima negati$\mathrm{va}^{19,23}$ e/ou (ii) exposição a algum tipo de arma (fogo e/ou branca). Ratificando, o adolescente que utiliza álcool e drogas ilícitas incorre em maior probabilidade de se expor à violência ${ }^{22}$.

Não foram considerados válidos os questionários em que o item sobre o uso de drogas e o CAGE (uso abusivo de álcool) não foram respondidos, apesar de os participantes terem referido acesso à arma e/ou ter resultado de autoestima negativa. Foram validados como não expostos os questionários que apresentaram respostas negativas para acesso à arma, afirmativas 


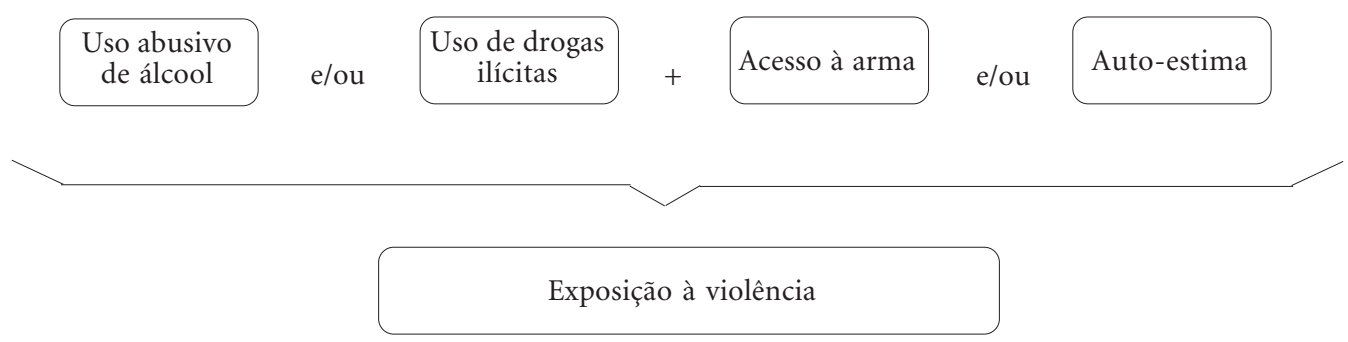

Figura 1. Representação da exposição do adolescente à violência.

Fonte: Dados da pesquisa.

para autoestima positiva, porém o item CAGE e uso de drogas ilícitas não foram preenchidos. A representação da exposição à violência exposta na Figura 1 demonstra as relações existentes entre os fatores de risco e seu desfecho.

Os dados foram digitados, organizados e tabulados no programa Statistical Package for the Social Sciences - SPSS, versão 15.0. Posteriormente, realizaram-se testes estatísticos descritivos, medidas de tendência central e dispersão. Para verificar a associação entre as variáveis utilizou-se o teste quiquadrado de Pearson $\left(\chi^{2}\right)$, num grau de confiabilidade de $95 \%$. O trabalho foi aprovado pelo Comitê de Ética da Universidade de Fortaleza e declara-se inexistir conflito de interesse.

\section{Resultados}

A média de idade dos participantes $(\mathrm{N}=458)$ foi de 14,4 anos, com desvio-padrão (DP) de $\pm 2,50$. Dentre as variáveis sociodemográficas e escolares prevaleceu o sexo masculino $(52,8 \%)$ e o período da adolescência entre 10 a 14 anos (55,2\%). Quanto à religião, predominou a católica $(62,4 \%)$, e em relação à naturalidade, a Região Metropolitana de Fortaleza preponderou (76,4\%).

Os dados identificaram que as famílias dos adolescentes sobrevivem com menos de um salário mínimo $(\mathrm{SM})(36,5 \%)$. No que concerne à escola, prevaleceu tempo de estudo $\leq 8$ anos $(78,6 \%)$, gostar de ir à escola $(69,3 \%)$ e se ausentar da mesma por mais de duas semanas nos últimos 6 meses $(58,3 \%)$, justificando esse absenteísmo $(n=273)$, por problemas de saúde $(35,9 \%)$ e familiares $(21,6 \%)$. Estas famílias englobavam quatro pessoas $(22,3 \%)$, a densidade demográfica, por domicílio, registrou 4,87 habitantes. A dinâmica familiar desse adolescente caracterizou-se por: residir com um responsável pela família $(53,5 \%)$ ou com os pais $(40,2 \%)$, ter pais separados $(53,3 \%)$, o responsável pela provisão da família ser os pais $(3,9 \%)$ e a inserção do provedor familiar no mercado de trabalho, formal ou informal $(67,0 \%)$.

Quanto às características familiares, prevaleceu a família monoparental/ampliada $(53,5 \%)$ e seguida da nuclear $(40,2 \%)$, o estado civil dos pais destacou a separação $(53,3 \%)$, o responsável pela provisão da família ser os pais $(3,9 \%)$ e o provedor familiar está inserido no mercado de trabalho, seja formal ou informal $(67,0 \%)$.

Os adolescentes referiram possuir pais etilistas $(32,3 \%)$ e terem relações familiares boas ou muito boas $(55,7 \%)$. Vale salientar que referiram a satisfação dos pais com o rendimento escolar $(69,7 \%)$ e com as amizades dos filhos $(52,4 \%)$ e, entre os participantes, predominou o consumo de bebidas alcoólicas nos últimos seis meses $(26,2 \%)$.

Conforme a análise do $\mathrm{CAGE}^{21}$, registrou-se os adolescentes que fizeram uso abusivo de álcool $(13,5 \%)$ e o alto risco para o uso abusivo (4,6\%). Além disso, identificou-se o uso de drogas ilícitas $(23,8 \%)$ e o acesso à arma $(26,9 \%)$. Ao avaliarmos a autoestima, prevaleceu uma forma positiva $(70,7 \%)$.

Considerando-se os fatores de risco à exposição à violência, encontrou-se que $17,7 \%$ ( $\mathrm{n}=$ $71 /$ casos válidos) estão expostos a este agravo (Tabela 1). Na associação observada entre variáveis sociodemográficas e escolares e a exposição à violência, prevaleceu o sexo masculino $(11,7 \%)$, período da adolescência entre 10 a 14 anos (9,2\%), 
Tabela 1. Distribuição do uso de álcool, drogas ilícitas, acesso à arma e autoestima entre adolescentes de uma comunidade de baixa renda. Fortaleza (CE), 2009. $(\mathrm{N}=458)$

\begin{tabular}{lrc}
\hline & $\mathbf{n}$ & $\%$ \\
\hline Álcool & & \\
$\quad$ Uso abusivo & 62 & 13,5 \\
Alto risco para uso abusivo & 21 & 4,6 \\
$\quad$ Negativo & 218 & 47,6 \\
$\quad$ Não respondeu & 157 & 34,3 \\
Uso drogas ilícitas & & \\
$\quad$ Sim & 109 & 23,8 \\
Não & 284 & 62,0 \\
Não respondeu & 65 & 14,2 \\
Acesso à arma & & \\
Sim & 123 & 26,9 \\
Não & 317 & 69,2 \\
Não respondeu & 18 & 3,9 \\
Autoestima (n $=437)^{*}$ & & \\
Positiva & 324 & 70,7 \\
Negativa & 113 & 24,7 \\
\hline
\end{tabular}

*Adolescentes que preencheram a escala de autoestima completa. católicos (10,1\%), naturais da Região Metropolitana de Fortaleza (12,0\%), renda salarial familiar menor que 2 SM $(18,2 \%)$, tempo de estudo $\leq$ 8 anos $(13,7 \%)$, gostarem de ir à escola $(12,1 \%)$ e o absenteísmo escolar (13,9\%) (Tabela 2).

Houve associação direta entre ser natural da Região Metropolitana de Fortaleza ( $p=0,020)$, absenteísmo escolar $(\mathrm{p}<0,001)$ e tempo de estudo $(\mathrm{p}=0,009)$ com a exposição à violência.

$\mathrm{Na}$ associação entre variáveis familiares e a exposição à violência, predominou famílias monoparental/ampliada $(10,1 \%)$, pais separados (11,2\%), responsável financeiro pela família sendo os pais $(0,8 \%)$, responsável trabalhando (12,3\%), pais ou responsáveis etilistas $(12,9 \%)$, relações familiares muito boas/boas (13,5\%), satisfação dos pais com rendimento escolar $(14,6 \%)$ e amizades $(8,4 \%)$ (Tabela 3$)$.

Encontrou-se associação direta entre o responsável financeiro pela família $(\mathrm{p}=0,026)$, pais ou responsáveis etilistas ( $\mathrm{p}<0,001)$, relações familiares $(p=0,009)$ e não satisfação dos pais com amizades dos filhos ( $\mathrm{p}<0,001)$ com a exposição à violência.

Tabela 2. Perfil sociodemográfico e escolar à exposição à violência entre adolescentes de uma comunidade de baixa renda. Fortaleza (CE), 2009.

\begin{tabular}{|c|c|c|c|c|c|}
\hline \multirow[b]{2}{*}{ Variáveis } & \multicolumn{2}{|c|}{ Exposto } & \multicolumn{2}{|c|}{ Não exposto } & \multirow[b]{2}{*}{$\mathbf{p}^{\star}$} \\
\hline & $\mathbf{n}$ & $\%$ & $\mathbf{n}$ & $\%$ & \\
\hline Sexo & & & & & 0,068 \\
\hline Masculino & 47 & 11,7 & 180 & 44,7 & \\
\hline Feminino & 24 & 6,0 & 151 & 37,6 & \\
\hline Período da adolescência (Em anos) & & & & & 0,981 \\
\hline 10 a 14 & 37 & 9,2 & 173 & 43,0 & \\
\hline 15 a 19 & 34 & 8,5 & 158 & 39,3 & \\
\hline Religião & & & & & 0,606 \\
\hline Católica & 40 & 10,1 & 210 & 52,9 & \\
\hline Outras & 28 & 7,1 & 119 & 30,0 & \\
\hline Naturalidade & & & & & 0,020 \\
\hline Região Metropolitana de Fortaleza & 48 & 12,0 & 264 & 66,0 & \\
\hline Outros municípios & 23 & 5,8 & 65 & 16,3 & \\
\hline Renda salarial familiar $\left(\mathrm{Em} \mathrm{SM}^{* *}\right)$ & & & & & 0,963 \\
\hline$<2$ & 61 & 18,2 & 250 & 74,4 & \\
\hline$\geq 2$ & 05 & 1,5 & 20 & 6,0 & \\
\hline Tempo de estudo (Em anos) & & & & & 0,009 \\
\hline$\leq 8$ & 54 & 13,7 & 256 & 65,0 & \\
\hline$>8$ & 15 & 3,8 & 69 & 17,5 & \\
\hline Gosta de ir à escola & & & & & 0,487 \\
\hline Sim & 47 & 12,1 & 232 & 59,5 & \\
\hline Não & 22 & 5,6 & 89 & 22,8 & \\
\hline Absenteísmo escolar ${ }^{* * *}$ & & & & & $<0,001$ \\
\hline Sim & 55 & 13,9 & 178 & 44,9 & \\
\hline Não & 13 & 3,3 & 150 & 37,9 & \\
\hline
\end{tabular}

${ }^{*}$ Teste do quiquadrado de Pearson; significativo quando $\mathrm{p}<0,05 .{ }^{* *} 1$ Salário Mínimo (SM), valor $=\mathrm{R} \$ 465,00 .{ }^{* *}$ Por mais de 2 semanas nos últimos 6 meses. 


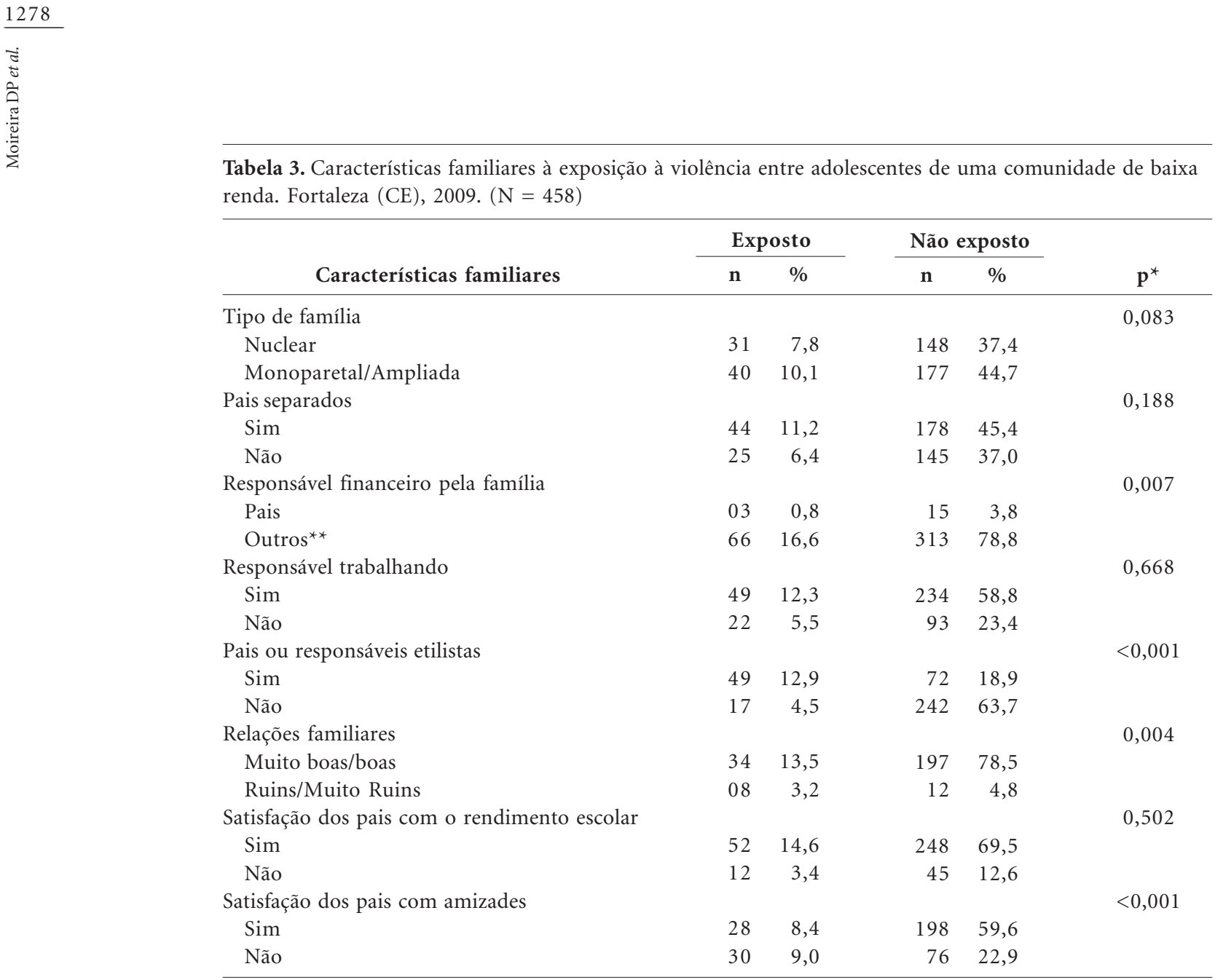

*Teste do quiquadrado de Pearson; significativo quando $\mathrm{p}<0,05 .{ }^{* *}$ Tios, avós, irmãos e pais.

\section{Discussão}

Importante destacar como limitação do estudo a dificuldade de ajuste de alguns fatores analisados e seu pertencimento aos níveis do modelo ecológico, em função da complexidade e polissemia com que o fenômeno se apresenta. Desta forma, as autoras justificam que nem todas as variáveis apresentadas estarão, necessariamente, inseridas nos níveis do modelo. Contudo esse fato não suprime a importância do estudo ao evidenciar as associações de alguns fatores com a exposição à violência no contexto comunitário.

Embora estudos demonstrem a associação entre a variável sexo e adolescentes em situações de vulnerabilidade social ${ }^{11,14,24}$ no que se refere à exposição à violência, o que não ocorreu nesta investigação.

As questões de gênero, aqui representadas pela variável sexo, suscitam reflexões. A sociedade perpetua as diferenças culturais na formação dos meninos e das meninas, reproduzidas pelo modo como as famílias conduzem a educação e a socialização dos seus filhos. A literatura reitera que os meninos, além de se envolverem com eventos violentos, enquanto agressores, também estão mais expostos a serem vítimas deles ${ }^{25}$. Os achados do estudo corroboram a literatura ao predominar o sexo masculino entre os adolescentes expostos à violência.

Existem evidências de uma continuidade no comportamento violento da infância à adolescência e da adolescência à fase adulta ${ }^{26}$, o que torna o adolescente mais vulnerável à violência devido a sua faixa etária ${ }^{27}$, pois a idade influencia sobre seu comportamento ${ }^{28}$. Dentre os resultados, o período compreendido entre 10 - 14 anos, apesar de não representar associação direta, prevaleceu entre os participantes $(\mathrm{p}=0,981)$.

No Brasil, sobretudo nas regiões metropolitanas e nos grandes centros urbanos, a violência tende a persistir e a ser utilizada como recurso por pessoas e grupos para conquistar mercados e poder ${ }^{14}$. Neste sentido, encontrou-se que ser da 
região metropolitana é fator de risco para a exposição à violência na comunidade, dado este registrado em outros estudos ${ }^{29,30}$.

Esta discussão pode estar respaldada pela dinâmica cotidiana dessas metrópoles, com ocorrências sistemáticas de festividades nos finais de semana, o deslocamento frequente entre cidades circunvizinhas, disputas de poder entre "tribos", status, maior disparidade entre as classes sociais, possibilitando conjunturas mais propicias às situações de violência ${ }^{31,32}$. Outra probabilidade explicativa é a expansão industrial ${ }^{33}$, e, com isso, maior concentração de pessoas e a ocorrência de eventos trabalhistas, culturais e mesmo científicos (congressos) que fomentam episódios que podem desencadear circunstâncias violentas.

Dentre os cenários de violência para o adolescente, identificamos a residência, a escola e suas redondezas. Um ambiente importante para a formação do adolescente é a escola, pois traz a tona discussões práticas acerca de sua formação e deve valorizar que a ternura e a cumplicidade solidária devem estar presentes nos atos e na comunidade $^{9}$. Estudo ${ }^{15}$ afirma o baixo nível de escolaridade (pouco tempo de estudo) entre jovens envolvidos em situações de violência. Nesta pesquisa, possuir pouco tempo ( $\leq 8$ anos) de estudo esteve associado à exposição da violência $(\mathrm{p}=0,009)$.

Os adolescentes que não gostam de ir à escola alegam desinteresse, conflitos, fracasso escolar, suspensão de aula e muitas vezes tendem ao abandono, isso representa uma dificuldade para as escolas mantê-los em sala de aula ${ }^{34}$. Diante dessa casuística, neste estudo, gostar de ir a escola é um fator protetor à exposição à violência $(\mathrm{p}=0,487)$.

As políticas públicas ${ }^{25,35}$ preconizam a importância da escola como um local singular de aglomeração adolescente, para diagnosticar situações limites que desvirtuem a sua cidadania, sugerindo a realização de atividades conjuntas entre escola, serviços de saúde, comunidades e famílias ${ }^{35}$.

Porém, ao se tratar de violência, ações voltadas para a prevenção deste agravo na adolescência devem ser gestadas e implantadas desde a primeira infância ${ }^{36}$, considerado o período da construção de valores. Durante a adolescência, estas "edificações” são amadurecidas, sendo o reflexo da formação que o mesmo colheu durante sua infância.

$\mathrm{O}$ ato de não gostar de ir a escola pode retratar, também, a situação em que as escolas públicas se encontram. Inexiste estrutura adequada, quadro docente de qualidade e satisfeito com suas atividades pedagógicas, condições mínimas de favorecer uma discussão fortalecida com um olhar na promoção da saúde. Esta assertiva é respaldada pela literatura ${ }^{37,38}$.
Neste estudo, a autoestima (considerada como fator analítico para a exposição à violência) referida pelos adolescentes mostrou-se positiva. Estudo demonstra que, quando envolvidos em situações de violência, os adolescentes consideram-se detentores de "poder", contribuindo para uma análise favorável de si mesmo ${ }^{39}$.

O esclarecimento da magnitude da autoestima em diversos contextos possibilita o conhecimento de um atributo considerável na elaboração de estratégias na saúde coletiva, para a prevenção de problemas no crescimento e desenvolvimento de adolescentes ${ }^{40}$. Dessa forma, se o conhecimento da autoestima permite a visualização do aspecto individual do adolescente, ações voltadas à promoção da saúde, abarcando as relações humanas e a sua interface com a eclosão da violência urge ser discutida pela sociedade.

No tocante às relações familiares, os resultados deste estudo apontaram que quanto melhor o relacionamento (referido pelo adolescente) com a sua família, maior a exposição à violência. De uma forma geral, ao se aludir a uma boa família, na concepção dos adolescentes pode estar implícita a ideia de liberdade permitida pela família.

A repreensão ou imposição de limites pode, em alguns momentos, não ser assimilada por esse grupo como favorável ao crescimento pessoal, pois este período pode caracterizar-se como um momento de crise vital, no qual anseiam por liberdade, negando a noção de limites.

Nesta pesquisa, houve ainda associação com a condição de o pai e/ou a mãe serem provedores da família com a exposição dos adolescentes à violência. Adverte-se que esta provisão remetese ao emprego/trabalho remunerado, não se relacionando com a demonstração e a verbalização dos vínculos afetivos desenvolvidos e próprios do âmago familiar. Destarte, uma das consequências desse trabalho fora do lar possa ser o favorecimento de longos períodos sem a presença dos pais, estando os adolescentes à mercê de suas próprias decisões e escolhas.

Ainda em relação ao meio familiar, os adolescentes que não residiam com os pais tornamse, significativamente, mais expostos à violência, sugerindo que a supervisão parental seja um importante aspecto de proteção ${ }^{24}$. Estudo realizado com meninos de escolas públicas na cidade de Pittsburgh (EUA) sugeriu que a relação parental autoritária e sem diálogo pode predispor o envolvimento do adolescente com a violência ${ }^{13}$.

Assim, a célula familiar não é a soma dos indivíduos. Esta possui características próprias (em virtude da individualidade) dos membros que a compõe, apresentam fatores e valores dis- 
tintos, distribuição de funções e papéis, liderança e laços afetivos, relações socioeconômicas e culturais que interferem no relacionamento, nas finanças, na estrutura ${ }^{41}$ e na dinâmica familiar.

O meio social (amizades, escolas e família) transmite ao adolescente o modelo de vida que influencia na expressão do seu comportamento. Isto porque, na vida cotidiana, o convívio social e intrafamiliar refletem no modo de ver e viver no mundo e na forma de encarar a vida ${ }^{42}$. Dentre a satisfação dos pais com o meio social do adolescente, os tipos de amizades associaram-se diretamente com a violência; o rendimento escolar não foi significante na análise.

No nível comunitário, a escola deve planejar ações que permitam a inserção e despertem a motivação desse grupo; perdê-lo desse contexto possibilita a exposição desses jovens à violência. Estudiosos $^{34,36}$ identificaram múltiplos fatores que favorecem a evasão escolar. Neste estudo, o absenteísmo escolar associou-se diretamente com a exposição à violência.

Causas que favorecem o absenteísmo escolar, a exemplo do consumo de drogas lícitas e ilícitas e o uso de armas de fogo, originam uma relação de mão dupla entre a violência e o adolescente. Ao mesmo tempo em que são usadas (droga e arma) para perpetrar atos infracionais, também se mostram na gênese das mortes de adolescentes ${ }^{9}$. Neste estudo, alguns adolescentes tiveram acesso às armas, ao uso do álcool e/ou ao consumo de drogas ilícitas.

$\mathrm{O}$ uso de armas brancas ou de fogo não representa por si um ato de violência, mas a expectativa de vivenciá-la ${ }^{10}$ prediz um comportamento de risco importante e uma atividade predominantemente masculina entre jovens em idade escolar ${ }^{26}$.

O álcool é um importante fator situacional que pode precipitar o envolvimento do adolescente com a violência ${ }^{25,26}$. Apesar de a lei brasileira proibir a venda de bebidas alcoólicas para menores de 18 anos $^{43}$, os adolescentes consomem álcool no convívio com amigos e familiares, em domicílio ou em ambiente público ${ }^{44}$. Em diversas circunstâncias, o uso de bebidas alcoólicas torna-se a porta de entrada para o uso abusivo e o início do consumo de drogas ilícitas ${ }^{45}$.

A literatura evoca que a qualidade do convívio existente entre os pais e os jovens pode ser determinante, tanto no envolvimento, quanto no aparecimento de desfechos decorrentes deste agravo $^{22}$, como o uso de álcool ${ }^{41}$, que, nesta pesquisa, apresentou associação direta entre exposição da violência e o adolescente dizer que os pais são etilistas ( $\mathrm{p}<0,001)$.

Corroborando a importância do contexto familiar como proteção ou risco para a violência, Horta et al. ${ }^{10}$ reafirmam que, atualmente, as famílias estão com diversos arranjos, facilitando a exposição do adolescente à violência.

Apesar de nesta investigação o tipo de organização da família $(\mathrm{p}=0,083)$ e ter pais separados $(\mathrm{p}=$ 0,188 ) não se associarem com a exposição do adolescente à violência, estas dimensões suscitam monitoramento e demandam novas pesquisas para o delineamento de estratégias preventivas, promotoras de saúde e transformação social.

Esta multideterminação acarreta desafios que se perpetuam entre conter o adolescente à exposição à violência e criar estratégias que o proteja, tornando-se foco das políticas públicas para proporcionar uma rede de apoio ao cuidado com o adolescente. Sinaliza ainda a desorganização social e a política dos grandes centros urbanos.

\section{Conclusão}

O estudo identificou, a partir da análise de características sociodemográficas, de familiares, da utilização de arma de fogo e/ou branca, do uso do álcool e/ou droga ilícita à exposição de adolescentes à violência, em uma comunidade de baixa renda, situada em capital do nordeste do Brasil.

Mostraram-se associados à exposição à violência os seguintes fatores: (i) os adolescentes apresentarem tempo de estudo menor ou igual a oito anos; (ii) absenteísmo escolar por mais de duas semanas nos últimos seis meses; (iii) possuírem pais responsáveis pelo sustento financeiro da família; (iv) seus pais ou responsáveis serem etilistas; (v) os adolescentes afirmarem que as relações familiares são boas/muito boas; (vi) a insatisfação dos pais com as amizades de seus filhos.

Diante desse cenário, reconhece-se a importância de se identificar esses fatores para subsidiar o planejamento em saúde e o desenvolvimento comunitário, no enfrentamento do problema. 


\section{Colaboradores}

DP Moreira, LJES Vieira e AMJ Pordeus participaram de todas as etapas do estudo. SVG Lira, GLM Luna e JG e Silva contribuíram com a interpretação dos dados e concepção final artigo. MFAS Machado colaborou na revisão crítica do artigo.

\section{Agradecimentos}

À Fundação Cearense de Apoio de Desenvolvimento Científico e Tecnológico - FUNCAP, ao Conselho Nacional de Desenvolvimento Científico e Tecnológico - CNPq (Bolsa Produtividade em Pesquisa) e à Coordenação de Aperfeiçoamento de Pessoal de Nível Superior - CAPES (Bolsa Mestrado).

\section{Referências}

1. Aisenberg E, Ell K. Contextualizing community violence and its effects. J Interpers Violence 2005; 20(7):7855-7871.

2. Deslandes SF, Souza ER, Minayo MCS, Costa CRBSF, Krempel M, Cavalcanti ML, Lima MLC, Moysés SJ, Leal ML, Carmos CN. Caracterização diagnóstica dos serviços que atendem vítimas de acidentes e violências em cinco capitais brasileiras. Cien Saude Colet 2007; 11(Supl. 1):1279-1290.

3. Waiselfisz JJ. Mapa da Violência: Os jovens da América Latina. Brasília, São Paulo: Rede de Informação Tecnológica Latino-Americana (RITLA), Ministério da Justiça, Instituto Sangari; 2008.

4. Brook JS, Brook DW, Whiteman M. Growing up in a violent society: longitudinal predictors of violence in Colombian adolescents. Am J Community Psychol 2007; 40(1-2):82-95.

5. Lopes RE, Adorno RCF, Malfitano APS, Takeiti BA, Silva CR, Borba PLO. Juventude pobre, violência e cidadania. Saude soc. 2008; 17(3):63-76.

6. Carvalho QCM, Cardoso MVLML, Silva MJ, Braga VAB, Galvão MTG. Violência contra criança e adolescente: reflexão sobre políticas públicas. Rev. RENE 2008; 9(2):157-164.

7. Silva KL, Dias FLA, Vieira NFC, Pinheiro PNC. Reflexões acerca do abuso de drogas e da violência na adolescência. Esc. Anna Nery 2010; 14(3):605610

8. Gudlaugsdottis GR, Vilhjalmsson R, Kristjansdottir G, Jacobsen R, Meyrowitsch D. Violent behaviour among adolescents in Iceland: a national survey. Int J Epidemiol 2004; 33(5):1046-1051.

9. Brasil. Ministério da Saúde (MS). Política Nacional de Promoção da Saúde. Brasília: MS; 2006.

10. Horta RL, Horta BL, Pinheiro RT, Krindges M. Comportamentos violentos de adolescentes e coabitação parento-filial. Rev Saude Publica 2010; 44(6):979-985.

11. Stafström M. Kick back and destroy the ride: Alcohol-related violence and associations with drinking patterns and delinquency in adolescence. Substance Abuse Treatment, Prevention, and Policy 2007; 18(18): 1-9.

12. Aredes RMP, Moares MS. Adolescentes em conflito com a lei. Cien Saude Colet 2007; 12(5):11851192.

13. Hoeve M, Blokland A, Dubas JS, Loeber R, Gerris JRM, Laan PH. Trajectories of Delinquency and Parenting Styles. J Abnorm Child Psychol 2008; 36(2):223-235.

14. Garcia BL, Freire TVM. O comportamento adolescente frente à violência delinquencial em uma comunidade de Fortaleza, Ceará. Fortaleza: UNIFOR; 2008.

15. Sena CA, Colares V. Comportamentos de risco para a saúde entre adolescentes em conflito com a lei. Cad Saude Publica 2008; 24(10):2314-2322.

16. Organização Mundial de Saúde (OMS). El embarazo y el aborto em la adolescencia. Washington: OMS; 1975.

17. Organização das Nações Unidas para a Educação, Ciência e Cultura (UNESCO) 2007. [página da Internet]. [acessado 2013 mar 24]. Disponível em: http://www.unesco.org/pt/brasilia 
18. Wright LM, Leahey M. Enfermeiras e Famílias: um guia para avaliação e intervenção na família. $4^{\mathrm{a}}$ Edição. São Paulo: Roca; 2008.

19. Avanci JQ, Assis SG, Santos NC, Oliveira RVC. Adaptação transcultural de escala de autoestima para adolescentes. Psicol. Reflex. Crit. 2007; 20(3):397-405.

20. Ceará. Secretaria da Saúde (SS). Não-violência: um desafio constante. Fortaleza: SS; 2003.

21. Masur J, Monteiro MG. Validation of the "CAGE" alcoholism screening test in a Brazilian psychiatric inpatient hospital setting. Braz. j. med. biol. Res 1983; 16(3):215-218.

22. Organização Mundial da Saúde (OMS). Relatório mundial sobre violência e saúde. Genebra: OMS; 2002.

23. Rosenberg M. Society and the adolescent self-image. Princeton: Princeton University Press; 1965.

24. Benetti SPC, Gama C, Vitolo M, Silva BS, D 'Ávila A, Zavaschi ML. Violência comunitária, exposição às drogas ilícitas e envolvimento com a lei na adolescência. Psico 2006; 37(3):276-286.

25. Brasil. Ministério da Saúde (MS). Marco Legal: saúde, um direito dos adolescentes. Brasília: MS; 2007.

26. Organización Panamericana de la Salud (OPAS). Informe mundial sobre la violencia y la salud. Washington: OPAS; 2002.

27. Souza MKB, Santana JSS. Atenção ao adolescente vítima de violência: participação de gestores municipais de saúde. Cien Saude Colet 2009; 14(2):547555.

28. Oliveira MT, Lima MLC, Barros MDA, Paz AM, Barbosa AMF, Leite RMB. Sub-registro da violência doméstica em adolescentes: a (in)visibilidade na demanda ambulatorial de um serviço de saúde no Recife-PE, Brasil. Rev. Bras. Saúde Matern. Infant. 2011; 11(1):29-39.

29. Lima MLC, Ximenes RAA, Feitosa CL, Souza ER, Albuquerque MFPM, Barros MDA, Souza WV, Lapa TM. Conglomerados de violência em Pernambuco, Brasil. Rev Panam Salud Publica 2005; 18(2):122-128.

30. Kodato S, Silva APS. Homicídios de Adolescentes: Refletindo sobre Alguns Fatores Associados. Psicol. Reflex. Crit. 2000; 13(3):507-515.

31. Banco Mundial (BM). Prevenção Comunitária do Crime e da Violência em Áreas Urbana da América Latina: um guia de recursos para municípios. Brasília: BM; 2003.

32. Hayeck CM. Refletindo sobre a violência. Rev. Bras. Hist Cien Soc 2009; 1:1-8.

33. Pereira JP. Direitos Humanos, Criminalidade e Capitalismo. Rev. Urutágua. 2007; 12(2):1-10.

34. Gallo AE, Williams LCA. A escola como fator de proteção à conduta infracional de adolescentes. Cad. Pesqui. 2008; 38(133):41-59.
35. Brasil. Ministério da Saúde (MS). Saúde Integral de Adolescentes e Jovens: Orientações para a Organização de Serviços de Saúde. Brasília: MS; 2007.

36. American Psychological Association (APA), The National Association for the Education of Young Children (NAEYC). Violence prevention in early childhood: how teachers can help. Washington: APA, NAEYC; 2002.

37. Paul JJ, Barbosa MLO. Qualidade docente e eficácia escolar. Tempo soc. 2008; 20(1):119-133.

38. Veiga L, Leite MRSDT, Duarte VC. Qualificação, Competência técnica e inovação no ofício docente para a melhoria da qualidade do ensino fundamental. Rev. adm. contemp. 2005; 9(3):143-167.

39. Chrispim LMD. Meninos que mataram: promoção de uma reintegração social saudável [dissertação]. Fortaleza: UNIFOR, 2005.

40. Avanci JQ, Assis SG, Santos NC, Oliveira RVC. Adaptação Transcultural de Escala de Auto Estima para adolescentes. Psicologia: Reflexão e Crítica 2007; 20(3): 397-405.

41. Brito HS. Estresse, resiliência e vulnerabilidade: comparando famílias com filhos adolescentes na escola. Rev Bras Crescimento Desenvolv Hum. 2006; 16(2):25-37.

42. Priuli RMA, Moraes MS. Adolescentes em conflito com a lei. Cien Saude Colet 2007; 12(5):1185-1192.

43. Brasil. Presidência da República. Lei 8.069, 13 jul. 1990. Dispõe sobre o Estatuto da Criança e do Adolescente e dá outras providências. Diário Oficial da União 1990; 16 jul.

44. Pratta EMM, Santos MA. Levantamento dos motivos dos responsáveis pelo primeiro contato com adolescentes do ensino médio com substâncias psicoativas. SMAD 2006; 2(2):1-17.

45. Cavalcante MBPT, Alves MDS, Barroso MGT. Adolescência, álcool e drogas: uma revisão na perspectiva da promoção da saúde. Esc Anna Nery Rev Enferm 2008; 12(3):555-559.

Artigo apresentado em 09/07/2012

Aprovado em 30/08/2012

Versão final apresentada em 24/09/2012 